\title{
State Estimation for Networked System with Random Delay Using Kalman Filter
}

\author{
Masaharu Yagi ${ }^{\dagger}$ and Yuichi Sawada ${ }^{\ddagger}$ \\ ${ }^{\dagger}$ Division of Engineering Design, Kyoto Institute of Technology \\ $\ddagger^{\ddagger}$ Division of Mechanical and System Engineering, Kyoto Institute of Technology \\ Matsugasaki, Sakyo, Kyoto 606-8585, Japan
}

E-mail: ${ }^{\dagger}$ d1821007@edu.kit.ac.jp, ${ }^{\ddagger}$ sawada@kit.ac.jp

\begin{abstract}
This paper discusses an approach of constructing a state estimator for a class of linear stochastic systems with randomly delayed observation data. State variables of the system is assumed to be observed over a communication network which causes the random delay. In our study, the random delay is assumed to consist of a sum of an average delay and a randomness. The observation data with the random delay can be represented by an observation model with state-dependent additive noise by using the Taylor series expansion around the time behind the average delay. This observation system becomes a stochastic bilinear system. The Kalman filter is constructed as the state estimator for the linear stochastic system based on the bilinear observation system. The effectiveness of the proposed Kalman filter is demonstrated for the several types of input signals such as a sinusoidal wave, a step function, a square wave and so on by numerical simulations.
\end{abstract}

\section{Introduction}

Recently, teleoperation technologies for robotic systems have gathered a lot of attention to provide human skills in the distant places. When it is considered that these technologies are introduced into general environments such as offices, homes, hospitals and so on, it is desirable that these are provided for people without special equipments. Therefore, to realize these goals, teleoperation technologies should be achieved by using existing communication networks such as local area networks (LAN), wide area networks (WAN) and wireless LANs. In use of the teleoperation systems, reference signals, state signals, observation signals are interacted with each other through the communication network. Thus, the networked systems are considered as the feedback systems with the communication network delay. Generally speaking, since the communication networks cause the random delay, those signals become noisy due to the random delay. Hence, it is very difficult that the state of the system is obtained and it is possible to cause the instability for the system. The state estimation problems for such systems with randomly delayed ob- servation have been investigated by a lot of researchers. Fan et al. studied a new scheme for the networked predictive control. This scheme mainly consists of the control prediction generator and network delay compensator [1]. Liu et al. considered the model predictive control problem for the closed-loop networked control systems. In Liu's research, a Markov chain is employed as the process of the random delay and the closed-loop networked control systems is represented as a Markovian jump system [2]. Xia et al. studied a networked predictive control scheme with the random delay modeled as an associated Markov chain [3]. The stochastic stability of their closed-loop networked predictive control systems was proved [3]. Wu et al. presented the mean square exponentially stability for the long random delay of networked systems [4]. Sun et al. studied the problem of robust stabilization for a class of uncertain networked systems with random delay. The system considered in Sun's research is described as a Markovian jump linear system with the random delay [5]. Guo et al. attempted a stochastic state feedback controller for the variable-period sampling networked systems with the network-induced time delay modeled by the uniform distribution [6]. Guo et al. considered a type of discrete-time system with the random delay and the exponentially stable in the mean square sense was proved [7]. Furthermore, they proposed filters for the networked control systems. Schenato researched about a design of the optimal estimators with the random delay and packet loss [8],[9]. Wang et al. accomplished to solve a filtering problem for linear uncertain discretetime stochastic systems with randomly varying sensor delay [10]. Moayedi et al. studied for adaptive filtering schemes for the discrete-time system with the random delays, packet dropouts and missing measurements [11]. In these researches, the Markov chain and so on were employed as the models of random delay. However, it is considered that these model for describing the random delay does not enough to represent the random delay, because these random delay is not employed the randomness, e.g. a white Gaussian noise.

In this paper, a Kalman filter for the networked systems with the random (transfer) delay is considered. Because the random delay considered here fluctuated 
around an average time delay, the sum of the average time delay and a white Gaussian noise is employed as the approximated random delay. Thus, the state signals, the observation signals and so on are affected by this random delay, when these signals reach to the other side over the communication network. To design the Kalman filter, a time-invariant linear system is defined as the remote system. The observation system includes the random delay. The Taylor series expansion form is applied to the observation system for treating the random delay explicitly in this study. As a result, the observation system with the random delay can be connected into an observation system with an additive a multiplicative noise defined by the product of the state and the randomness. Hence, the Kalman filter is designed for the time-invariant linear system and the observation with the multiplicative noise. When this filter is constructed for estimating the state of the system, a Lyapunov equation of the state is required. Therefore, three equations are needed to reduce the effect of the random delay such as the estimate equation of the system, the Ricatti equation for the estimation error covariance and the Lyapunov equation for the state. The effectiveness of the proposed Kalman filter is confirmed by the numerical simulations. When the numerical simulations for its effectiveness, several kinds of reference inputs are employed.

\section{Problem Statement}

In this paper, a state estimation problem for the following time-invariant linear stochastic system with the random delay is investigated:

$$
\begin{aligned}
& d x(t)=A x(t) d t+B u(t) d t+G d w_{s}(t) \\
& d y(t)=C x\left(t-\left(T+\gamma_{o}(t)\right)\right) d t, \quad\left(T+\gamma_{o}(t)>0\right)
\end{aligned}
$$

where $x(t) \in \Re^{n}$ and $y(t) \in \Re^{m}$ represent the state vector and the observation vector, respectively. The random delay, $T+\gamma_{o}(t)$, is greater than zero and the other conditions of the random delay are described in detail afterward. In this system, $u(t) \in \Re^{1}$ denotes a control input. $d w_{s}(t)$ indicates an increment of Wiener process with zero-mean and its covariance $Q_{s}\left(:=\mathcal{E}\left\{d w_{s}^{2}(t)\right\}\right)$. Furthermore, $d w_{s}(t)$ is defined as $\gamma_{s}(t) d t$, i.e. $d w_{s}(t):=\gamma_{s}(t)$ where $\gamma_{s}(t)$ is the system noise which is defined as a white Gaussian noise. On the other hand, in Eq. (2), $T$ is an average time delay and $\gamma_{o}(t)$ is a random noise defined by

$$
\gamma_{o}(t)= \begin{cases}\gamma(t) & \text { for } \gamma_{o}(t)>-T \\ 0 & \text { otherwise }\end{cases}
$$

where $\gamma(t)$ denotes the white Gaussian noise whose mean and covariance are $\mathcal{E}\{\gamma(t)\}=0$ and $\mathcal{E}\left\{\gamma^{2}(t)\right\}=$ : $R$, respectively. Hence, the random delay can be described by the sum of these values, i.e. $T+\gamma_{o}(t)$. Assuming that the covariance of $\gamma(t)$ holds $R \ll T^{2}$, the randomness of the random delay $\gamma_{o}(t)$ can be approximated by the white Gaussian noise $\gamma(t)$, i.e. $\gamma_{o}(t) \cong$ $\gamma(t)$. Under this assumption, $\gamma_{o}(t)$ is regarded on the white Gaussian noise $\left(\mathcal{E}\left\{\gamma_{o}^{2}(t)\right\}=R\right)$ for simplicity in this paper. Now, $T$ is assumed to be known and the system noise, $\gamma_{s}(t)$ and the noise for the random delay, $\gamma_{o}(t)$ are assumed to be uncorrelated variables, i.e. $\mathcal{E}\left\{\gamma_{s}(t) \gamma_{o}(t)\right\}=0$. Furthermore, $T+\gamma_{o}(t)$ must be the positive value because this is the random delay, or Eq. (2) calculates the states in the future. Therefore, to calculate the states as the random delay system, $\gamma_{o}(t)$ is assumed to be greater than $-T$. In the following discussion, the randomness of the random delay, $\gamma_{o}(t)$ is replaced into $\gamma(t)$ for the above reasons.

To treat the random delay explicitly, the Taylor series expansion form is applied to state vector $x(t-(T+\gamma(t)))$ in the observation system around $t_{s}(:=t-T)$. Then, Eq. (2) is rewritten as the undermentioned form:

$$
d y(t) \cong C x\left(t_{s}\right) d t-C \gamma(t) d x\left(t_{s}\right)
$$

where the high order terms with respect to $d t$ are neglected. Substituting Eq. (1) into Eq. (4), we obtain

$$
\begin{aligned}
d y(t) \cong & C x\left(t_{s}\right) d t-C\left\{A x\left(t_{s}\right)+B u\left(t_{s}\right)\right\} d w_{o}(t) \\
& -C G \gamma_{s}\left(t_{s}\right) \gamma(t) d t .
\end{aligned}
$$

As seen in the right-hand side of Eq. (5), the product of the noises, $\gamma_{s}\left(t_{s}\right)$ and $\gamma(t)$ appears. However, because these noises are uncorrelated random variables each other, the corresponding term can be ignored. Thus, eventually, the observation equation can be approximated as the following equation:

$$
d y(t)=C x\left(t_{s}\right) d t-C\left\{A x\left(t_{s}\right)+B u\left(t_{s}\right)\right\} d w_{o}(t),
$$

where $d w_{o}(t)(:=\gamma(t) d t)$ represents an increment of Wiener process with zero-mean and its covariance $R(:=$ $\left.\mathcal{E}\left\{d w_{o}^{2}(t)\right\}\right)$. The second term of the right-hand side of this equation behaves as the multiplicative noise in order to obtain the information of $x\left(t_{s}\right)$. Therefore, a state estimator for this system should be designed for reducing the multiplicative noise. In the next section, a Kalman filter is designed as the state estimator.

\section{Synthesis of Kalman Filter}

The Kalman filter is designed for the stochastic system expressed by Eqs. (1) and (6). In constructing the Kalman filter, because $\gamma(t)$ has the small variance, $T+\gamma(t)$ almost becomes the positive value. Therefore, the assumption for $\gamma(t)$ given by Eq. (3) is not considered, First of all, an innovation process, $d \nu(t)$ is defined as

$$
\begin{aligned}
d \nu(t) & =d y(t)-C \hat{x}\left(t_{s} \mid t_{s}\right) d t \\
& =C e\left(t_{s}\right) d t-C\left\{A x\left(t_{s}\right)+B u\left(t_{s}\right)\right\} d w_{o}(t),
\end{aligned}
$$


where $\hat{x}\left(t_{s} \mid t_{s}\right)$ denotes the state estimate; and $e\left(t_{s}\right):=$ $x\left(t_{s}\right)-\hat{x}\left(t_{s}\right)$. The correlation function of $d \nu(t)$ can be calculated as follows:

$$
\begin{aligned}
\mathcal{E}\{ & \left.d \nu(t) d \nu^{\mathrm{T}}(t)\right\} \\
=\mathcal{E}\{ & {\left[C e\left(t_{s}\right) d t-C\left\{A x\left(t_{s}\right)+B u\left(t_{s}\right)\right\} d w_{o}(t)\right] } \\
& \left.\times\left[C e\left(t_{s}\right) d t-C\left\{A x\left(t_{s}\right)+B u\left(t_{s}\right)\right\} d w_{o}(t)\right]^{\mathrm{T}}\right\} \\
= & C \mathcal{E}\left\{\left\{A x\left(t_{s}\right)+B u\left(t_{s}\right)\right\}\left\{A x\left(t_{s}\right)+B u\left(t_{s}\right)\right\}^{\mathrm{T}}\right\} R C^{\mathrm{T}} \\
= & C\left[A \mathcal{E}\left\{x\left(t_{s}\right) x^{\mathrm{T}}\left(t_{s}\right)\right\} A^{\mathrm{T}}+B \mathcal{E}\left\{u\left(t_{s}\right) x^{\mathrm{T}}\left(t_{s}\right)\right\} A^{\mathrm{T}}\right. \\
& \left.+A \mathcal{E}\left\{x\left(t_{s}\right) u^{\mathrm{T}}\left(t_{s}\right)\right\} B^{\mathrm{T}}+B \mathcal{E}\left\{u\left(t_{s}\right) u^{\mathrm{T}}\left(t_{s}\right)\right\} B^{\mathrm{T}}\right] R C^{\mathrm{T}} \\
= & C\left[A \Pi\left(t_{s}\right) A^{\mathrm{T}}+B Q_{u} B^{\mathrm{T}}\right] R C^{\mathrm{T}},
\end{aligned}
$$

where $\Pi\left(t_{s}\right)$ denotes the autocorrelation matrix value of the state, i.e. $\Pi\left(t_{s}\right)=\mathcal{E}\left\{x\left(t_{s}\right) x^{\mathrm{T}}\left(t_{s}\right)\right\}$; and $Q_{u}$ denotes the autocorrelation value of the input, i.e. $Q_{u}=\mathcal{E}\left\{u^{2}\left(t_{s}\right)\right\}$. In this procedure, the state, $x\left(t_{s}\right)$ and the input, $u\left(t_{s}\right)$ are assumed to be uncorrelated variables each other. Thus, the correlation functions of these variable becomes zero, i.e. $\mathcal{E}\left\{x\left(t_{s}\right) u^{\mathrm{T}}\left(t_{s}\right)\right\}=$ $\mathcal{E}\left\{u\left(t_{s}\right) x^{\mathrm{T}}\left(t_{s}\right)\right\}=0$.

Then, from Eq. (8), the state estimate, $\hat{x}\left(t_{s} \mid t_{s}\right)$ can be derived by using the orthogonal projection [12]; the estimation error covariance, $P\left(t_{s} \mid t_{s}\right)$ is given by the following Ricatti differential equation; and the autocorrelation function of the state, $\Pi\left(t_{s}\right)$ can be calculated as the Lyapunov differential equation. Therefore, the equations of $\hat{x}\left(t_{s} \mid t_{s}\right), P\left(t_{s} \mid t_{s}\right)$ and $\Pi\left(t_{s}\right)$ are represented by

$$
\begin{aligned}
& d \hat{x}\left(t_{s} \mid t_{s}\right)=A \hat{x}\left(t_{s} \mid t_{s}\right) d t+B u\left(t_{s}\right) d t \\
& +P\left(t_{s} \mid t_{s}\right) C^{\mathrm{T}}\left[C\left\{A \Pi\left(t_{s}\right) A^{\mathrm{T}}+B Q_{u} B^{\mathrm{T}}\right\} R C^{\mathrm{T}}\right]^{-1} \\
& \times\left\{d y(t)-C \hat{x}\left(t_{s} \mid t_{s}\right) d t\right\} \\
& \dot{P}\left(t_{s} \mid t_{s}\right)=A P\left(t_{s} \mid t_{s}\right)+P\left(t_{s} \mid t_{s}\right) A^{\mathrm{T}}+G Q_{s} G^{\mathrm{T}} \\
& -P\left(t_{s} \mid t_{s}\right) C^{\mathrm{T}}\left[C\left\{A \Pi\left(t_{s}\right) A^{\mathrm{T}}+B Q_{u} B^{\mathrm{T}}\right\} R C^{\mathrm{T}}\right]^{-1} \\
& \times C P\left(t_{s} \mid t_{s}\right) \\
& \dot{\Pi}\left(t_{s}\right)=A \Pi\left(t_{s}\right)+\Pi\left(t_{s}\right) A^{\mathrm{T}}+G Q_{s} G^{\mathrm{T}} .
\end{aligned}
$$

Now, because the input signal is also sent through the communication network, $u\left(t_{s}\right)$ can not be obtained. Hence, the input signal, $u\left(t_{s}\right)$ in Eq. (9) is replaced into $u\left(t-\left(T+\gamma_{o}(t)\right)\right):$

$$
\begin{aligned}
& d \hat{x}\left(t_{s} \mid t_{s}\right)=A \hat{x}\left(t_{s} \mid t_{s}\right) d t+B u(t-(T+\gamma(t))) d t \\
&+P\left(t_{s} \mid t_{s}\right) C^{\mathrm{T}}\left[C\left\{A \Pi\left(t_{s}\right) A^{\mathrm{T}}+B Q_{u} B^{\mathrm{T}}\right\} R C^{\mathrm{T}}\right]^{-1} \\
& \quad \times\left\{d y(t)-C \hat{x}\left(t_{s} \mid t_{s}\right) d t\right\} .
\end{aligned}
$$

\section{Numerical Simulation Setup}

The numerical simulations are performed to demonstrate the effectiveness of the proposed Kalman filter.

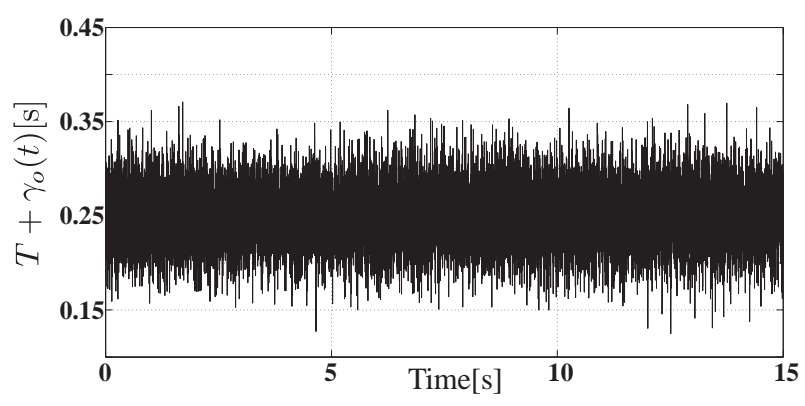

Fig. 1: Random delay, $T+\gamma_{o}(t)$.

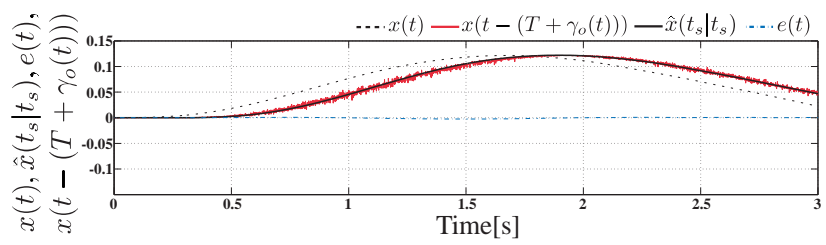

(a) $x(t), x\left(t-\left(T+\gamma_{o}(t)\right)\right), \hat{x}\left(t_{s} \mid t_{s}\right)$ and $e(t)$.

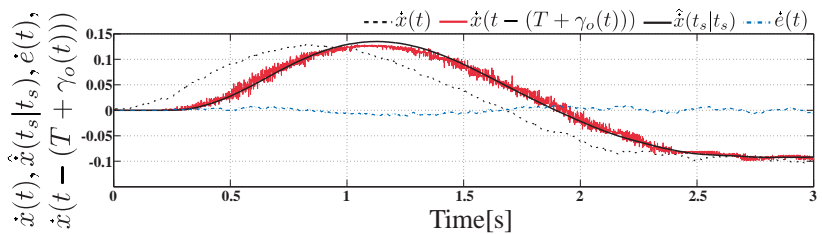

(b) $\dot{x}(t), \dot{x}\left(t-\left(T+\gamma_{o}(t)\right)\right), \hat{\dot{x}}\left(t_{s} \mid t_{s}\right)$ and $\dot{e}(t)$.

Fig. 2: Numerical simulation results (Input: Sinusoidal wave, frequency: $1.0[\mathrm{rad} / \mathrm{sec}])$.

In this numerical simulations, the second-order system is employed as the example system expressed by

$$
\begin{aligned}
d x(t) & =A x(t) d t+B u(t) d t+G d w_{s}(t) \\
d y(t) & =C x\left(t-\left(T+\gamma_{o}(t)\right)\right) d t,
\end{aligned}
$$

where

$$
\begin{aligned}
& A=\left[\begin{array}{cc}
0 & 1 \\
-10 & -1
\end{array}\right], B=\left[\begin{array}{l}
0 \\
1
\end{array}\right], G=\left[\begin{array}{c}
0 \\
0.5
\end{array}\right], C=\left[\begin{array}{ll}
1 & 0 \\
0 & 1
\end{array}\right] \\
& Q_{u}=1.0, R=0.01 .
\end{aligned}
$$

The average time delay for the random delay, $T$ is set as 0.25 , i.e. $T=0.25[\mathrm{~s}]$.

To confirm the performance of the proposed Kalman filter, the input signal for the system equation, $u(t)$ is set by the several input signals such as the sinusoidal wave, the step function, the square wave and the triangle wave.

\section{$5 \quad$ Numerical Results}

Fig. 1 shows the random delay process, $T+\gamma_{o}(t)$, modeled by the sum of the average time delay, $T$ and the white Gaussian noise, $\gamma_{o}(t)$. Fig. 2 shows the 


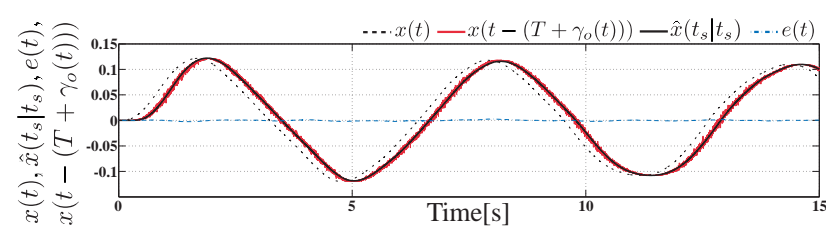

(a) $x(t), x\left(t-\left(T+\gamma_{o}(t)\right)\right), \hat{x}\left(t_{s} \mid t_{s}\right)$ and $e(t)$.

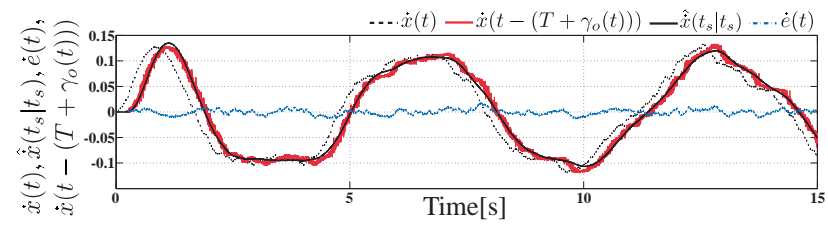

(b) $\dot{x}(t), \dot{x}\left(t-\left(T+\gamma_{o}(t)\right)\right), \hat{\dot{x}}\left(t_{s} \mid t_{s}\right)$ and $\dot{e}(t)$.

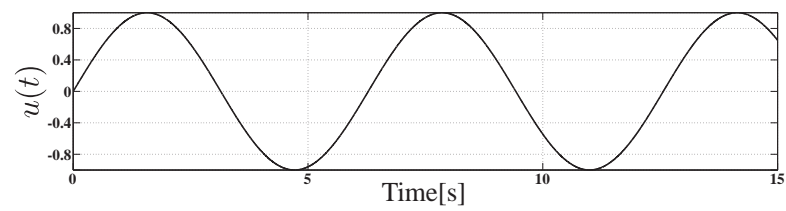

(c) $u(t)$.

Fig. 3: Numerical simulation results (Input: Sinusoidal wave, frequency: $1.0[\mathrm{rad} / \mathrm{sec}])$.

magnified figure of the numerical simulation which is demonstrated by using the sinusoidal wave. Furthermore, Figs. 3 to 7 show numerical simulation results which are performed by using the sinusoidal wave withe the low frequency, the step function, the square wave, the triangle wave and the sinusoidal wave with the high frequency, respectively. In all figures, the black dashed line indicates the state, $x(t)$ and its derivative, $\dot{x}(t)$; the red solid line indicates the observation state, $x\left(t-\left(T+\gamma_{o}(t)\right)\right)$ and $\dot{x}\left(t-\left(T+\gamma_{o}(t)\right)\right)$; the black solid line indicates the state estimate, $\hat{x}\left(t_{s} \mid t_{s}\right)$ and $\hat{\dot{x}}\left(t_{s} \mid t_{s}\right)$; and the blue dashed-dotted line indicates the error, $e(t)\left(:=x\left(t_{s}\right)-\hat{x}\left(t_{s}\right)\right)$ and $\dot{e}(t)\left(:=\dot{x}\left(t_{s}\right)-\hat{\dot{x}}\left(t_{s}\right)\right)$. Furthermore, (c) of each figures show the input signal applied to the system, $u(t)$.

As seen in Fig. 2, because the system has the system noise modeled by the white Gaussian noise, $x(t)$ and $\dot{x}(t)$ are affected by the system noise. Furthermore, by comparing the states at the current time, $x(t)$ and $\dot{x}(t)$ with the states through the communication network, $x\left(t-\left(T+\gamma_{o}(t)\right)\right)$ and $\dot{x}\left(t-\left(T+\gamma_{o}(t)\right)\right)$, it is found that the states, $x(t)$ and $\dot{x}(t)$ are vibrated more violently. Hence, in the proposed system, $x(t)$ and $\dot{x}(t)$ are affected by the system noise and the random delay, $T+\gamma_{o}(t)$ (shown in Fig. 1). Since the system over the communication network can only obtain the states through the network such as $x\left(t-\left(T+\gamma_{o}(t)\right)\right)$ and $\dot{x}\left(t-\left(T+\gamma_{o}(t)\right)\right)$, these states cause the instability when in use for control of the system. Thus, the fluctuation caused by the system noise and the random delay must be reduced.

To do this aim, the performance of the proposed

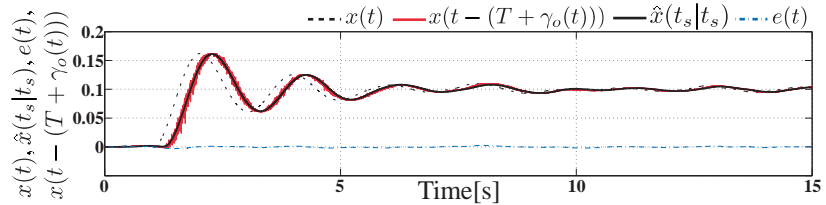

(a) $x(t), x\left(t-\left(T+\gamma_{o}(t)\right)\right), \hat{x}\left(t_{s} \mid t_{s}\right)$ and $e(t)$.

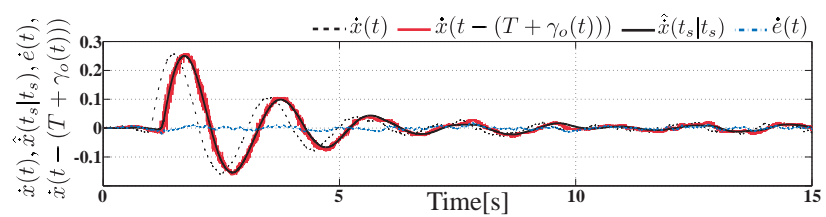

(b) $\dot{x}(t), \dot{x}\left(t-\left(T+\gamma_{o}(t)\right)\right), \hat{\dot{x}}\left(t_{s} \mid t_{s}\right)$ and $\dot{e}(t)$.

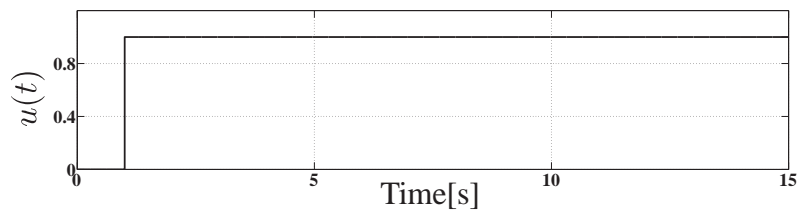

(c) $u(t)$.

Fig. 4: Numerical simulation results (input: Step function).

Kalman filter is discussed below. As previously mentioned, The effectiveness of the system noise and the random delay should be reduced. First of all, as seen in Figs. 3 to 7 , by comparing the state estimates, $\hat{x}\left(t_{s} \mid t_{s}\right)$ and $\hat{\dot{x}}\left(t_{s} \mid t_{s}\right)$ with the states through the network, $x\left(t-\left(T+\gamma_{o}(t)\right)\right)$ and $\dot{x}\left(t-\left(T+\gamma_{o}(t)\right)\right)$, it is found that $\hat{x}\left(t_{s} \mid t_{s}\right)$ and $\hat{\dot{x}}\left(t_{s} \mid t_{s}\right)$ behave as the smoothed $x\left(t-\left(T+\gamma_{o}(t)\right)\right)$ and the smoothed $\dot{x}\left(t-\left(T+\gamma_{o}(t)\right)\right)$. Thus, it is said that the proposed Kalman filter can achieve to reduce the effectiveness of the random delay. Second, from another point of view, by comparing the state estimates, $\hat{x}\left(t_{s} \mid t_{s}\right)$ and $\hat{\dot{x}}\left(t_{s} \mid t_{s}\right)$ with the states at the current time, $x(t)$ and $\dot{x}(t)$, it is also found that $\hat{x}\left(t_{s} \mid t_{s}\right)$ and $\hat{\dot{x}}\left(t_{s} \mid t_{s}\right)$ behave as the states which are smoothed $x(t)$ and $\dot{x}(t)$, and $\hat{x}\left(t_{s} \mid t_{s}\right)$ and $\hat{\dot{x}}\left(t_{s} \mid t_{s}\right)$ become the states which are delayed $x(t)$ and $\dot{x}(t)$ by the average time delay, $T$. Hence, it is also said that our Kalman filter can realize to reduce the effectiveness of the system noise. Furthermore, in Figs. 3 to 7 , because the error and the differentiated error, $e(t)$ and $\dot{e}(t)$ are obviously very small, it is considered that the accuracy for estimating is well. Therefore, for the above reasons, the Kalman filter which is designed in this paper is well-designed.

On the other hand, Fig. 7 shows the simulation result, when the input signal is used as the sinusoidal wave. In this case, the frequency of this sinusoidal wave is greater than the frequency of the previous sinusoidal wave which is used in Fig. 3. As seen in the result shown in Fig. 7 , the state estimates, $\hat{x}\left(t_{s} \mid t_{s}\right)$ and $\hat{\dot{x}}\left(t_{s} \mid t_{s}\right)$ can be enough estimated as is the case with the result of the previous sinusoidal wave. 


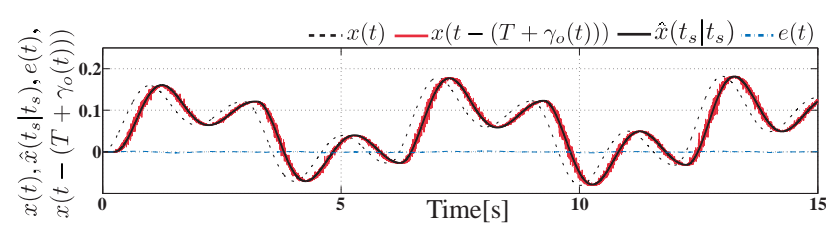

(a) $x(t), x\left(t-\left(T+\gamma_{o}(t)\right)\right), \hat{x}\left(t_{s} \mid t_{s}\right)$ and $e(t)$.

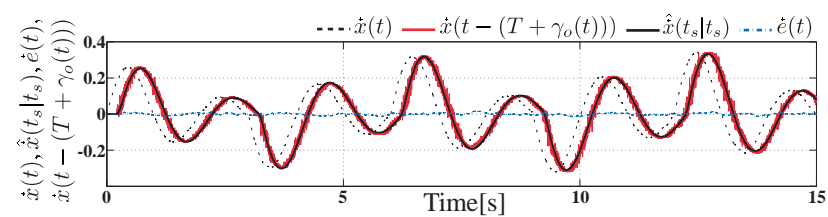

(b) $\dot{x}(t), \dot{x}\left(t-\left(T+\gamma_{o}(t)\right)\right), \hat{\dot{x}}\left(t_{s} \mid t_{s}\right)$ and $\dot{e}(t)$.

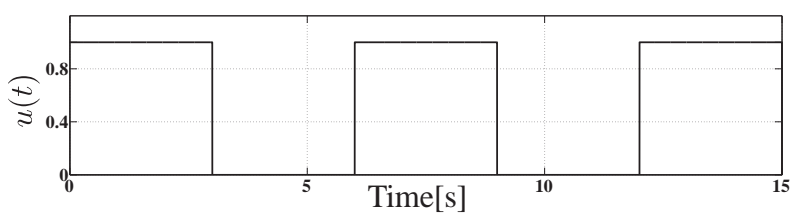

(c) $u(t)$.

Fig. 5: Numerical simulation results (input: Square wave).

By summarizing the above discussions, regardless of the class of the input signals and the frequency, it is said that the proposed Kalman filter designed in this research can reduce the effectiveness of the system noise and the random delay which are defined as the white Gaussian noise and the sum of the average time delay and the white Gaussian noise, $T+\gamma_{o}(t)$, respectively.

\section{Conclusions}

This paper discussed the state estimation problem for networked control systems with the random delay. It was considered that the random delay consists of the average time delay and the randomness in our research. The random delay was modeled as a sum of the average time delay and the white Gaussian noise and the constant time delay was assumed to be known. Then, the time-invariant stochastic linear system and the observation system were defined for designing the Kalman filter. In this research, the time-invariant stochastic linear system had the system noise which was employed as the white Gaussian noise. On the other hand, the observation noise did not include for the sake of simplicity to solve this problem. Furthermore, to treat the random delay explicitly, the Taylor series expansion form was applied to the observation system and the high order terms were ignored. As a result, the approximated observation system had the multiplicative noise which consisted of the product of the states and the randomness of the random delay. The Kalman filter was designed for these systems based on the innovation process. The state estimator including the multiplica-

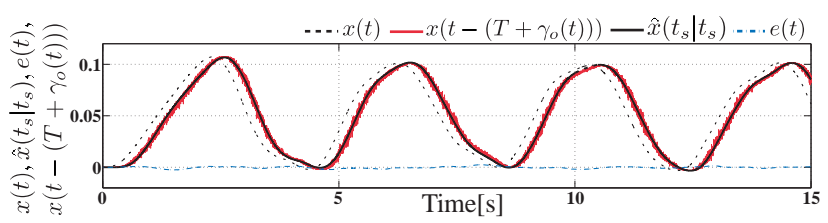

(a) $x(t), x\left(t-\left(T+\gamma_{o}(t)\right)\right), \hat{x}\left(t_{s} \mid t_{s}\right)$ and $e(t)$.

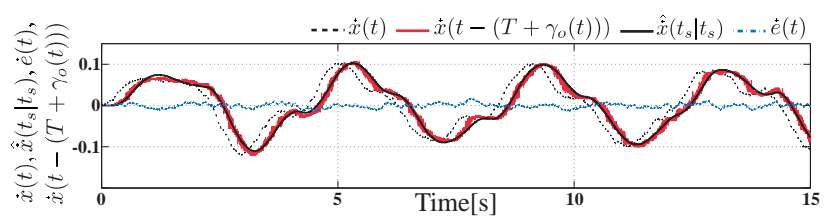

(b) $\dot{x}(t), \dot{x}\left(t-\left(T+\gamma_{o}(t)\right)\right), \hat{\dot{x}}\left(t_{s} \mid t_{s}\right)$ and $\dot{e}(t)$.

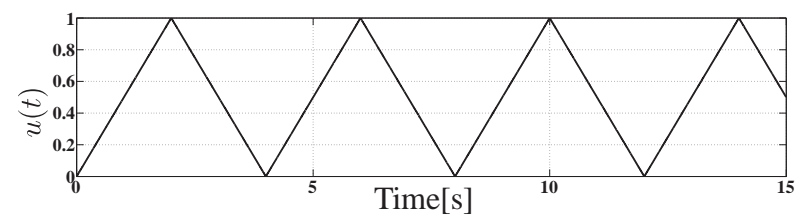

(c) $u(t)$.

Fig. 6: Numerical simulation results (input: Triangle wave).

tive noise, the Lyapunov differential equation for the state is needed to estimate the state value. Therefore, it was found that three equations were required to estimate the states with the average time delay such as the estimate state system, the Ricatti equation for the estimation error covariance and the Lyapunov differential equation for the autocorrelation of the states. To confirm the performance of the proposed Kalman filter, the numerical simulations were performed for several types of inputs such as the sinusoidal wave with the low frequency, the step function, the square wave, the triangle wave and the sinusoidal wave withe the high frequency. As seen in the results of the numerical simulations, the proposed Kalman filter could reduce the system noise and the randomness of the random delay regardless of the kinds of the input signal and the value of the frequency. Therefore, the Kalman filter designed in this research was well-designed for the networked control system with the random delay.

\section{Acknowledgment}

This work was supported by Japan Society for the Promotion of Science (JSPS) Grant-in-Aid for Scientific Research (C)25420184.

\section{References}

[1] Y. Fan, S. Liu and X. Liu: A New Predictive Control Systems with Network Delays in the Feedback 


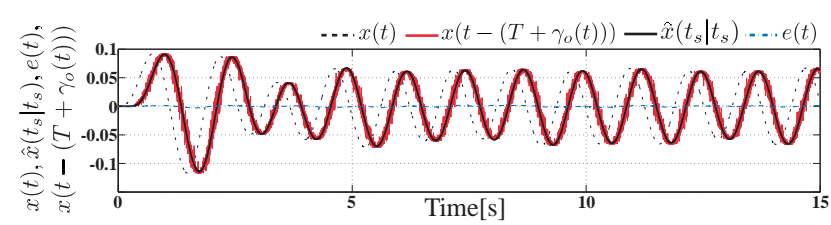

(a) $x(t), x\left(t-\left(T+\gamma_{o}(t)\right)\right), \hat{x}\left(t_{s} \mid t_{s}\right)$ and $e(t)$.

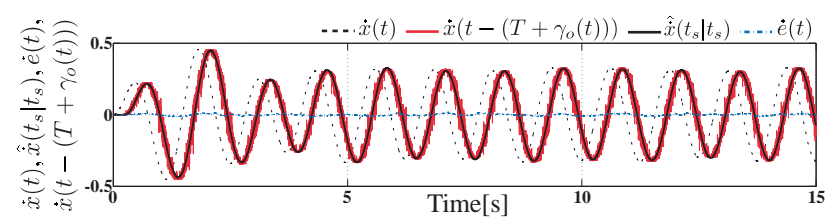

(b) $\dot{x}(t), \dot{x}\left(t-\left(T+\gamma_{o}(t)\right)\right), \hat{\dot{x}}\left(t_{s} \mid t_{s}\right)$ and $\dot{e}(t)$.

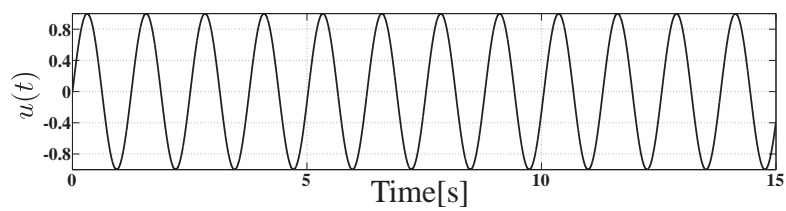

(c) $u(t)$.

Fig. 7: Numerical simulation results (Input: Sinusoidal wave, frequency: $5.0[\mathrm{rad} / \mathrm{sec}])$.

and Forward Channels, 2011 Chinese Control and Decision Conference, pp.912-916, 2011.

[2] A. Liu, L. Yu, W.-A. Zhang and D. He: Model Predictive Control for Networked Control System with Random Delay and Packet Disordering, Proceedings of 2011 8th Asian Control Conference, pp.653-658, 2011.

[3] Y. Xia, G.-P. Liu, M. Fu and D. Rees: Predictive control of networked systems with random delay and data dropout, IET Control Theory and Applications, 3 11, pp.1476-1486, 2009.

[4] J. Wu and F. Deng: Control of Long Random Delay Networked Control Systems with Prescribed Degree of Stability, Proceedings of the 6th World Congress on Intelligent Control and Automation, 1, pp.1270-1274, 2006.

[5] Y. Sun and Y. Huo: Robust static output feedback control for networked control system with random time delay, Conference on 2nd International Advanced Computer Control, 3, pp.547-551, 2010.

[6] X. Guo and H. Wang: Stochastic optimal control based on variable-period sampling model for Networked Control Systems with random delays, 2009 Chinese Control and Decision Conference, pp.5582-5586, 2009.

[7] L. Guo and H. Gu: Robust Stability of Discrete Systems with Uncertainties and Random Delay,
2010 International Conference on Measuring Technology and Mechatronics Automation, 3, pp.291294, 2010.

[8] L. Schenato: Kalman Filtering for Networked Control Systems with Random Delay and Packet Loss, 17th International Symposium on Mathematical Theory of Networks and Systems, 2006.

[9] L. Schenato: Optimal Estimation in Networked Control Systems Subject to Random Delay and Packet Drop, IEEE Transactions on Automatic Control, 53 5, pp.1311-1317, 2008.

[10] Z. Wang and D. W. C. Ho: Robust filtering under randomly varying sensor delay with variance constraints, IEEE Transactions on Circuits and Systems II: Express Briefs, 51 6, pp.230-326, 2004.

[11] M. Moayedi, Y. K. Foo and Y. C. Soh: Adaptive Kalman Filtering in Networked Systems With Random Sensor Delays, Multiple Packet Dropouts and Missing Measurements, IEEE Transactions on Signal Processing, 58 3, pp.1577-1588, 2010.

[12] X. Lu, H. Wang and X. Wang: On Kalman Smoothing for Wireless Sensor Networks Systems with Multiplicative Noises, Journal of Applied Mathematics, 2012, 19 pages, 2012. 